\title{
Identification of key genes in non-alcoholic fatty liver disease progression based on bioinformatics analysis
}

\author{
LIN LI ${ }^{1,2}$, HUABAO LIU $^{1}$, XIAOYU HU ${ }^{2}$, YI HUANG $^{1}$, YANAN WANG $^{1}$, YANSHA HE $^{1}$ and $^{\text {QINGSONG LEI }}{ }^{3}$ \\ ${ }^{1}$ Department of Liver Disease, Chongqing Traditional Chinese Medicine Hospital, Chongqing 400021; \\ ${ }^{2}$ Department of Infectious Disease, Affiliated Hospital of Chengdu University of Traditional Chinese Medicine, \\ Chengdu, Sichuan 610072; ${ }^{3}$ Department of Infectious Disease, The First Affiliated Hospital of \\ Chongqing Medical University, Chongqing 400016, P.R. China
}

Received October 19, 2017; Accepted February 22, 2018

DOI: $10.3892 / \mathrm{mmr} .2018 .8852$

\begin{abstract}
Due to economic development and lifestyle changes, the incidence of non-alcoholic fatty liver disease (NAFLD) has gradually increased in recent years. However, the pathogenesis of NAFLD is not yet fully understood. To identify candidate genes that contribute to the development and progression of NAFLD, two microarray datasets were downloaded from the Gene Expression Omnibus database. The differentially expressed genes (DEGs) were identified and functional enrichment analyses were performed. A protein-protein interaction network was constructed and modules were extracted using the Search Tool for the Retrieval of Interacting Genes and Cytoscape. The enriched functions and pathways of the DEGs included 'cellular macromolecule biosynthetic process', 'cellular response to chemical stimulus', 'extracellular matrix organization', 'metabolic pathways', 'insulin resistance' and 'forkhead box protein O1 signaling pathway'. The DEGs, including type-1 angiotensin II receptor, formin-binding protein 1-like, RNA-binding protein with serine-rich domain 1, Ras-related C3 botulinum toxin substrate 1 and polyubiquitin- $\mathrm{C}$, were identified using multiple bioinformatics methods and validated in vitro with reverse transcription-quantitative polymerase chain reaction analysis. In conclusion, five hub genes were identified in the present study, and they may aid
\end{abstract}

Correspondence to: Mr. Huabao Liu, Department of Liver Disease, Chongqing Traditional Chinese Medicine Hospital, 6 Seventh Branch of Panxi Road, Jiangbei, Chongqing 400021, P.R. China

E-mail: 15909386658@163.com

Abbreviations: BP, biological process; DEGs, differentially expressed genes; GEO, Gene Expression Omnibus; GO, Gene Ontology; KEGG, Kyoto Encyclopedia of Genes and Genomes; HC, healthy control; MF, molecular function; NAFLD, non-alcoholic fatty liver disease; NASH, non-alcoholic steatohepatitis; PPI, protein-protein interaction; SS, steatosis

Key words: differentially expressed genes, microarray, insulin resistance, non-alcoholic fatty liver disease, protein-protein interaction in understanding of the molecular mechanisms underlying the development and progression of NAFLD.

\section{Introduction}

Nonalcoholic fatty liver disease (NAFLD), one of the most common hepatic conditions, is closely associated with obesity, type II diabetes and insulin resistance. NAFLD encompasses multiple pathological events, including steatosis, non-alcoholic steatohepatitis (NASH) and cirrhosis, in addition to end-stage liver disease $(1,2)$. In the past, NAFLD has been more common in Western countries, although the incidence of NAFLD has increased in the Asian-Pacific region in recent years (3). Notably, the prevalence of NAFLD amongst younger populations is increasing. NAFLD poses a great threat to human health and society and is a important challenge in the liver disease field.

The development of NAFLD is the result of a combination of genetic, environmental and metabolic factors. At present, the most widely accepted theory of NAFLD pathogenesis is the two-hits theory, in which lipid accumulation in hepatocytes triggers a series of cytotoxic events that subsequently induce liver inflammation. Numerous pathological processes are induced, including insulin resistance, leptin deficiency, oxidative stress, fat accumulation and liver tissue inflammation (4). Accumulating evidence from previous studies examining the genetic and metabolic factors of NAFLD pathogenesis have demonstrated that the abnormal expression or mutation of key genes leads to the development and progression of NAFLD. These genes include signal transducer and activator of transcription 3 (STAT3), Krueppel-like factor 6 (KLF6), peroxisome proliferator-activated receptors (PPARs) and methylenetetrahydrofolate reductase (MTHFR), in addition to various pro-inflammatory cytokines (5). The interleukin (IL)-6 receptor subunit $\beta$-STAT3 signaling pathway is activated in NAFLD and may result in increased insulin resistance (6). KLF6 may mediate glucose tolerance and insulin response in NAFLD. Furthermore, KLF6 mediates PPAR $\alpha$ signaling induction (7,8). Gene polymorphisms (C677T and A1298C) of MTHFR are reported to be closely associated with susceptibility to NAFLD $(9,10)$. However, the exact mechanisms remain unclear due to the complex pathogenesis of this disease. 
Therefore, it is crucial to understand the precise molecular mechanisms involved in the development and progression of NAFLD in order to develop and evaluate effective diagnostic or management strategies.

In the present study, two mRNA microarray datasets from the Gene Expression Omnibus (GEO) were downloaded and analyzed to identify differentially expressed genes (DEGs) among healthy control (HC), steatosis (SS) and NASH groups. Gene Ontology (GO), Kyoto Encyclopedia of Genes and Genomes (KEGG) pathway enrichment and protein-protein interaction (PPI) network analyses were performed to understand the potential molecular mechanisms of fat accumulation and inflammation in hepatic cells. A total of five hub genes were identified, which may be candidate diagnostic or therapeutic biomarkers for NAFLD.

\section{Materials and methods}

Data analysis. The gene expression datasets GSE33814 and GSE89632 were downloaded from the GEO (www.ncbi.nlm.nih.gov/geo) (11) database. Samples in the above datasets were divided into three groups: SS vs. HC, NASH vs. HC, and NASH vs. SS. Detailed parameters of the datasets are presented in Table I.

Microarray data were submitted to the online database repository GEO2R (www.ncbi.nlm.nih.gov/geo/geo2r) to identify DEGs among the groups. GEO2R is an interactional and accessible online tool that allows investigators to compare two or more datasets and obtain a list of DEGs in GEO series across different experimental conditions. GEO2R, as well as the GEO query and Limma packages in R, were used to analyze the original microarray data (12). The distribution of the data was visualized using a box-and-whisker plot. A log fold change of $>0.3$ and an adjusted P-value of $<0.05$ was considered to indicate a statistically significant difference. Probe sets without corresponding gene symbols or genes with more than one probe set were removed or averaged, respectively. Subsequently, genes that matched the screening criteria in both datasets were overlapped in a Venn diagram in order to obtain the candidate DEGs.

Functional enrichment analyses of the DEGs. The Database for Annotation, Visualization and Integrated Discovery (DAVID; version 6.8; david.ncifcrf.gov) is an online biological information database that integrates biological data and analysis tools. DAVID provides a comprehensive set of functional annotation information on genes and proteins for users to extract biological information (13). To analyze the function of the DEGs, functional annotation and biological analyses were performed using DAVID. $\mathrm{P}<0.05$ was considered to indicate a statistically significant result.

Construction of PPI networks and analysis of modules. The DEGs were imported into the Search Tool for the Retrieval of Interacting Genes (STRING; version 10.0; string-db.org) online database for PPI network construction (14). Genes without connections to other genes were removed. Interactions with a combined score of $>0.9$ were considered statistically significant. PPI networks were drawn using Cytoscape (version 3.5.1), an open source bioinformatics software platform for visualizing molecular interaction networks (15). The size of nodes represents the degree of one gene. The degree of one gene means the number of its interactions with other genes. The higher the degree of one gene, the more important it is in the PPI network. Different colored nodes represent different groups. The top three modules in the PPI networks were identified using the Cytoscape plug-in app multicontrast delayed enhancement (MCODE) (16). The criteria for selecting modules were as follows: MCODE score, >5; degree cutoff, 10; node score cutoff, 0.2 ; and k-score, 2.

Prediction of the candidate microRNAs (miRNAs/miRs), drugs and diseases associated with the identified DEGs. An analysis to identify the KEGG pathways, miRNAs, drugs and diseases associated with the hub genes in these modules was performed using the WEB-based Gene SeT AnaLysis Toolkit (Webgestalt; www.webgestalt.org). Webgestalt is an online tool used to analyze functional enrichment in gene sets and is an integrated system for data mining, including KEGG pathways, GO, miRNAs, drugs and diseases (17). $\mathrm{P}<0.05$ was considered to indicate a statistically significant result.

Cell culture, construction of cell model and validation of hub genes in vitro. To validate the results of the bioinformatics analysis, the expression of hub genes was assessed in vitro. The human hepatic cell line LO2 was derived from the primary culture of human normal liver cells via continuous culture in vitro and is capable of continuous passage. $\mathrm{LO} 2$ cell growth and death is very similar to that of normal liver cells. Compared with HepG2 cells, LO2 are more sensitive to lipid metabolism. The cell line is widely used to assess the molecular effects of genes or drugs on fatty liver cells in vitro $(18,19)$. LO2 cells were bought from Chongqing Bopei Biotechnology Co., Ltd. (Chongqing, China; www.mengbio.com) and were preserved in the Chongqing Key Laboratory of Infectious Diseases and Parasitic Disease (Chongqing, China). Cells were cultured in Dulbecco's modified Eagle's medium (Gibco; Thermo Fisher Scientific, Inc., Waltham, MA, USA) supplemented with $10 \%$ fetal bovine serum (Gibco; Thermo Fisher Scientific, Inc.) and $1 \%$ penicillin/streptomycin at $37^{\circ} \mathrm{C}$ in a $5 \% \mathrm{CO}_{2}$ incubator until cells reached $90 \%$ confluence. Cells were subsequently seeded in a 6 -well plate $\left(5 \times 10^{4}\right.$ cells $\left./ \mathrm{ml}\right)$ and incubated for $24 \mathrm{~h}$ prior to treatment with $200 \mu \mathrm{M}$ oleic acid (OA; Sigma-Aldrich; Merck KGaA Darmstadt, Germany) in fatty acid-free 5\% bovine serum albumin (BSA; Sigma-Aldrich; Merck KGaA; pre-warmed to $50^{\circ} \mathrm{C}$ ) for $24 \mathrm{~h}$ to model steatotic cells. Control cells were treated with fatty acid free 5\% BSA without OA. Cells were subsequently harvested for quantification.

RNA extraction and reverse transcription-quantitative polymerase chain reaction $(R T-q P C R)$. Total RNA was isolated using TRIzol reagent (Invitrogen; Thermo Fisher Scientific, Inc.) and further purified using an RNeasy Mini kit (Qiagen GmbH, Hilden, Germany), according to the manufacturer's protocol. The concentration and quality of RNA was verified by spectrophotometry (NanoDrop 2000; Thermo Fisher Scientific, Inc.). cDNA was synthesized using a PrimeScript ${ }^{\mathrm{TM}}$ RT Reagent kit (Takara Biotechnology Co., Ltd., Dalian, China) according to the manufacturer's protocol. qPCR was performed using a LightCycler system (Roche 
Table I. Details of the non-alcoholic fatty liver disease datasets retrieved from the GEO database.

\begin{tabular}{lllcccc}
\hline Author, year & GEO dataset & \multicolumn{1}{c}{ Platform } & No. of samples & No. of cases & Organism & (Refs.) \\
\hline Starmann, 2012 & \multirow{2}{*}{ GSE33814 } & Illumina HumanWG-6 v3.0 & 44 & HC, 13 & Homo sapiens & (2) \\
& & expression beadchip & & SS, 19 & & \\
& & & & NASH, 12 & & \\
Arendt, 2015 & GSE89632 & Illumina HumanHT-12 WG-DASL & 63 & HC, 24 & Homo sapiens & (4) \\
& & V4.0 R2 expression beadchip & & SS, 20 & & \\
& & & & NASH, 19 & & \\
\hline
\end{tabular}

GEO, Gene Expression Omnibus; HC, healthy control; SS, steatosis; NASH, non-alcoholic steatohepatitis.

Diagnostics, Basel, Switzerland) in a volume of $10 \mu \mathrm{l}$ with the SYBR Premix Ex Taq ${ }^{\mathrm{TM}}$ II kit (Takara Biotechnology Co., Ltd.). The gene-specific primers were purchased from Sangon Biotech Co., Ltd. (Shanghai, China) and the sequences were as follows: Type-1 angiotensin II receptor (AGTR1) forward, 5'-TAAGATTGCTTCAGCCAGCGTCAG-3' and reverse, 5'-GAACAATAGCCAGGTATCGATCAATGC-3'; formin-binding protein 1-like (FNBP1L) forward, 5'-AGA CCATGAATA ACATTGACCGCCTAC-3' and reverse, 5'-ACTGCTATGTCTTCTGTCTCCTCTCC-3'; RNA-binding protein with serine-rich domain 1 (RNPS1) forward, 5'-GCT GAAGCACATGGATGGAGGAC-3' and reverse, 5'-GGC GGTGGTGGCAACATTCTC-3'; Ras-related C3 botulinum toxin substrate 1 (RAC1) forward, 5'-AGACAAGCCGATTGC CGATGTG-3' and reverse, 5'-TGCCGCACCTCAGGATAC CAC-3'; polyubiquitin-C (UBC) forward, 5'-TGGTGCTCC GTCTCAGAGGTG-3' and reverse, 5'-TCTGCTGGTCAG GAGGAATGCC-3'; $\beta$-actin forward, 5'-GCAAGCAGGAAC GATGAG-3' and reverse, 5'-CCATGCCAATGTTGTCTC TT-3'. The thermocycling conditions were as follows: $95^{\circ} \mathrm{C}$ for $10 \mathrm{~min}$, followed by 40 cycles of $95^{\circ} \mathrm{C}$ for $15 \mathrm{sec}$ and $58^{\circ} \mathrm{C}$ for $30 \mathrm{sec}$. Gene expression was analyzed using the comparative $2^{-\Delta \Delta \mathrm{Cq}}$ method and normalized to the internal reference gene $\beta$-actin (20). All experiments were performed in triplicate and data are presented as mean \pm standard deviation.

Statistical analysis. Statistical analysis was performed using SPSS software (version 19.0; IBM Corp., Armonk, NY, USA). For comparisons between two groups, Student's two tailed t-test was used. Data are presented as the mean \pm standard deviation. $\mathrm{P}<0.05$ was considered to indicate a statistically significant difference.

\section{Results}

Identification of DEGs for diagnosis or treatment of NAFLD. By normalization and analysis of the microarray datasets, a number of DEGs were identified in GSE33814 and GSE89632 (Table II). The DEGs from these groups were overlapped in a Venn diagram and 190 DEGs were subsequently identified in the SS vs. HC group (Fig. 1A); 1,106 genes were identified in the NASH vs. HC group (Fig. 1B); and 34 genes were identified in the NASH vs. SS group (Fig. 1C). Notably, by comparing DEGs in these groups, two DEGs were identified in all three groups: Immunoglobulin $\gamma$ Fc region receptor II-a (FCGR2A) and La-related protein 1B (LARP1B). Thus, these genes may be considered as specific diagnostic markers for NAFLD (Fig. 1D). A total of 107 DEGs overlapped in the SS vs. HC and NASH vs. HC groups, which may be involved in the advanced phase of disease progression. A total of 25 DEGs were overlapped in the NASH vs. SS and NASH vs. HC groups, which may be associated with the severity of NAFLD. Additionally, a total of 81 unique DEGs were identified in the SS vs. HC group, which may be associated with the onset of NAFLD. Furthermore, 972 unique DEGs were identified only in the NASH vs. HC group, which may be downstream of the 107 DEGs identified in the SS vs. HC and NASH vs. HC groups. A total of seven unique DEGs were identified in the NASH vs. SS group, which may not serve important roles in the progression of NAFLD. No DEGs overlapped in the SS vs. $\mathrm{HC}$ and NASH vs. SS groups (Fig. 1D).

Functional enrichment analysis and KEGG pathways of DEGs. To analyze the biological classification of the DEGs, functional and pathway enrichment analyses were performed using the functional enrichment analysis web tool DAVID. $\mathrm{GO}$ analysis revealed that genes in the SS vs. HC group were significantly enriched in cytosol $\left(\mathrm{P}=8.93 \times 10^{-3}\right)$, ion binding $\left(\mathrm{P}=3.85 \times 10^{-2}\right)$ and RNA binding $\left(\mathrm{P}=6.03 \times 10^{-3}\right)$ terms. The biological process (BP) analysis revealed that the DEGs were significantly enriched in the following processes: 'Cellular macromolecule biosynthetic process' $\left(\mathrm{P}=1.60 \times 10^{-2}\right)$, 'glycerophospholipid biosynthetic process' $\left(\mathrm{P}=3.60 \times 10^{-2}\right)$ and 'programmed cell death' ( $\mathrm{P}=4.64 \times 10^{-2}$; Fig. 2A).

DEGs in the NASH vs. HC group were significantly enriched in 'extracellular region' $\left(\mathrm{P}=1.05 \times 10^{-2}\right)$, 'carbohydrate derivative binding' $\left(\mathrm{P}=1.65 \times 10^{-3}\right)$ and 'enzyme binding' $\left(\mathrm{P}=5.69 \times 10^{-6}\right)$. BP analysis revealed that the DEGs were significantly enriched in the following processes: 'Regulation of signaling' $\left(\mathrm{P}=1.01 \times 10^{-7}\right)$, 'cellular response to chemical stimulus' $\left(\mathrm{P}=2.20 \times 10^{-9}\right)$ and 'response to organic substances' $\left(\mathrm{P}=5.34 \times 10^{-8}\right.$; Fig. 2B).

DEGs in the NASH vs. SS group were significantly enriched in 'extracellular region' $\left(\mathrm{P}=2.23 \times 10^{-4}\right)$ and 'exopeptidase activity' $\left(\mathrm{P}=2.33 \times 10^{-2}\right)$. BP analysis demonstrated that the DEGs were significantly enriched in processes associated with extracellular matrix organization $\left(\mathrm{P}=3.63 \times 10^{-5}\right)$, 'regulation of phosphate metabolic process' $\left(\mathrm{P}=9.55 \times 10^{-3}\right)$ and 
Table II. DEGs in microarray datasets GSE33814 and GSE89632.

\begin{tabular}{lccc}
\hline Comparison group & Microarray dataset & No. of DEGs & No. of overlapped genes \\
\hline SS vs. HC & GSE33814 & 766 & 190 \\
& GSE89632 & 5,381 & 1,106 \\
NASH vs. HC & GSE33814 & 3,656 & 5,607 \\
NASH vs. SS & GSE89632 & 1,328 & 34 \\
& GSE33814 & 147 & \\
\hline
\end{tabular}

DEG, differentially expressed gene; HC, healthy control; SS, steatosis; NASH, non-alcoholic steatohepatitis.
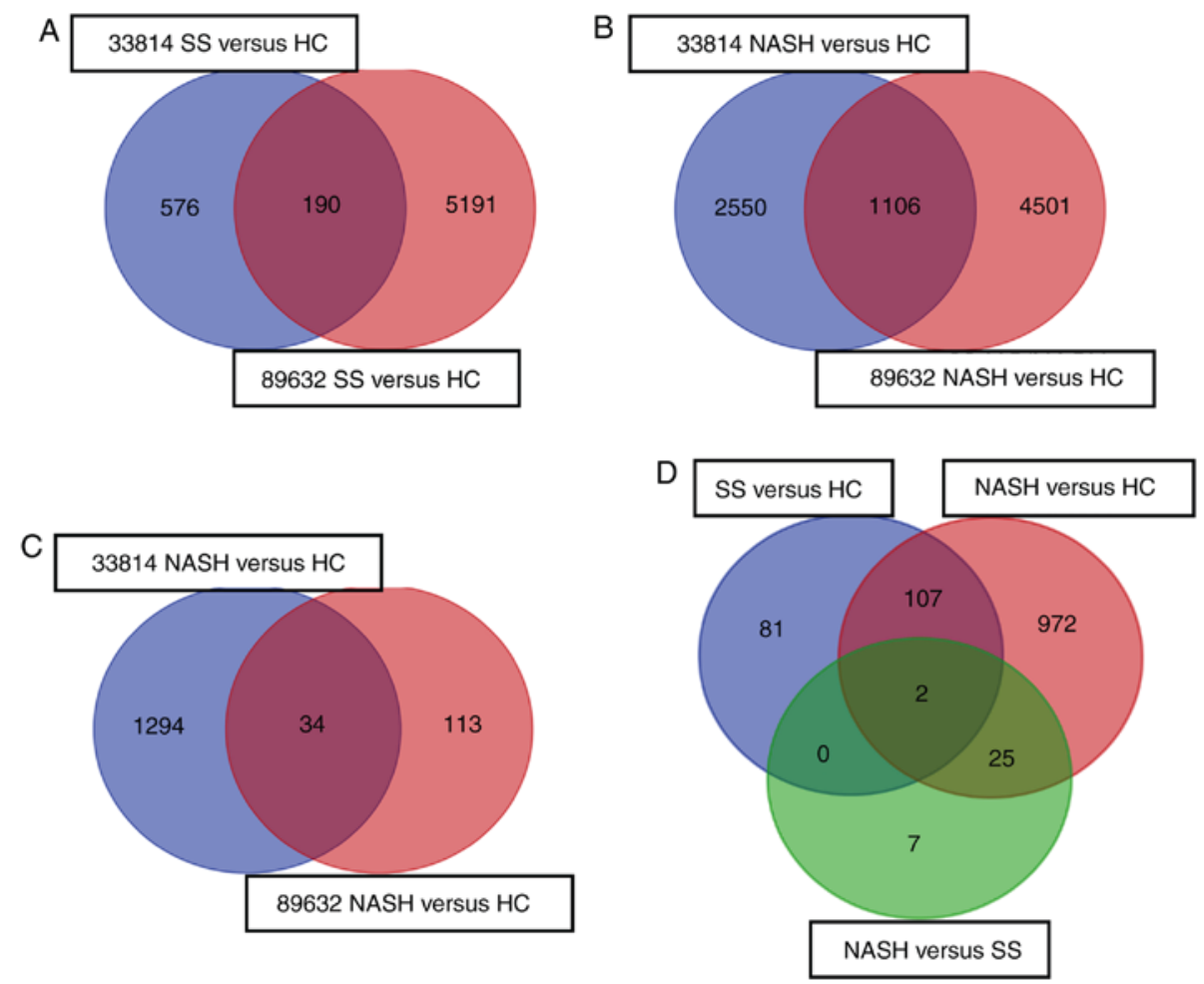

Figure 1. DEGs were identified in the mRNA expression profiles GSE33814 and GSE89632. The DEGs in different comparison groups were selected using a Venn diagram. DEGs were compared in the (A) SS vs. HC group, (B) NASH vs. HC group, (C) NASH vs. SS group and (D) between the three comparison groups. DEG, differentially expressed gene; SS, steatosis; HC, healthy control; NASH, non-alcoholic steatohepatitis.

'regulation of catalytic activity' $\left(\mathrm{P}=2.96 \times 10^{-2}\right)$. The above results indicated that during the early stages of NAFLD, alterations in biosynthetic processes were predominantly identified in hepatocytes. In the advanced stages of NAFLD, alterations in lipid metabolism and responses to stimuli may have crucial involvement in the progression of the disease (Fig. 2C).

The significantly enriched KEGG pathways were additionally identified at a significance level of $\mathrm{P}<0.05$ using DAVID. The results revealed that pathways in the SS vs. HC group were significantly enriched in metabolic pathways $\left(\mathrm{P}=4.17 \times 10^{-2}\right)$, cyclic guanosine monophosphate (cGMP)-protein kinase $\mathrm{G}$ (PKG) signaling pathway $\left(\mathrm{P}=4.48 \times 10^{-2}\right)$ and transcriptional misregulation in cancer $\left(\mathrm{P}=4.67 \times 10^{-2}\right.$; Fig. $\left.2 \mathrm{~A}\right)$. Pathways in the NASH vs. HC group were significantly enriched in the $5^{\prime}$ adenosine monophosphate-activated protein kinase
(AMPK) signaling pathway $\left(\mathrm{P}=2.29 \times 10^{-4}\right)$, insulin resistance $\left(\mathrm{P}=3.79 \times 10^{-4}\right)$ and forkhead box protein $\mathrm{O} 1$ (FoxO) signaling pathway $\left(\mathrm{P}=4.70 \times 10^{-3}\right.$; Fig. $\left.2 \mathrm{~B}\right)$. Pathways in the NASH vs. SS group were significantly enriched in extracellular matrix (ECM)-receptor interaction ( $\mathrm{P}=2.82 \times 10^{-2}$; Fig. 2C). Consistent with the results of GO analysis, KEGG analysis revealed that DEGs in the early stage of NAFLD were predominantly enriched in the metabolism of numerous substances, whereas DEGs involved in the advanced stages were predominantly enriched in lipid metabolism and its subsequent effect on target organs.

Construction of PPI networks. Following the removal of isolated genes, PPI networks were constructed using STRING and Cytoscape. The PPI network contained 493 nodes and 1,412 edges in the NASH vs. HC group (Fig. 3A); 37 nodes 

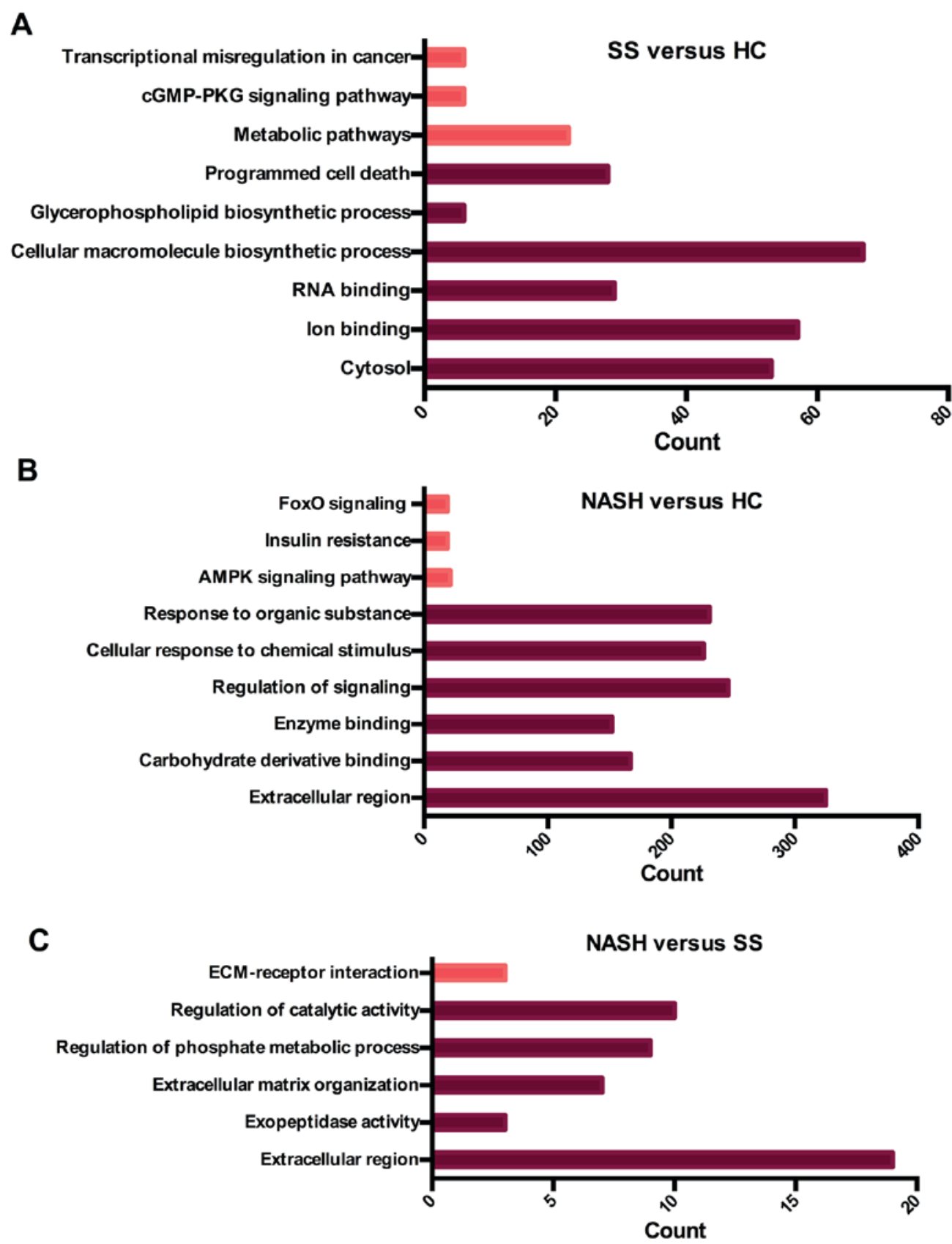

Figure 2. Summary of the top GO and KEGG results of DEGs. (A) Results of SS vs. HC group. (B) Results of NASH vs. HC group. (C) Results of NASH vs. SS group. The count represents the number of genes that enriched in one GO or KEGG term. The deep red bars represent GO in terms of DEGs. The light red bars represent KEGG in terms of DEGs. DEG, differentially expressed gene; SS, steatosis; HC, healthy control; NASH, non-alcoholic steatohepatitis; KEGG, Kyoto Encyclopedia of Genes and Genomes; GO, gene ontology; ECM, extracellular matrix; FoxoO, Forkhead box protein O1; cGMP-PKG; cyclic guanine monophosphate-dependent protein kinase, isozyme 1; AMPK, 5'-AMP-activated protein kinase.

and 36 edges in the SS vs. HC group (Fig. 3B); and two nodes and one edge in the NASH vs. SS group (Fig. 3C).

Following this, the indexes of betweenness centrality and degree were used to analyze the topological properties of the $\mathrm{SS}$ vs. HC and the NASH vs. HC groups, respectively. The top $70 \%$ genes were selected by these two measures for subsequent analyses. A total of 34 unique genes in the SS vs. HC group and 424 genes in NASH vs. HC groups were obtained. The top three genes in the SS vs. HC group were RNPS1, vesicle-associated membrane 3 and AGTR1. The top three genes in the NASH vs. HC group were UBC, phosphatidylinositol 3-kinase regulatory subunit $\alpha$ and $\mathrm{RACl}$. In parallel, there were 16 genes that overlapped in the SS vs. HC and the NASH vs. HC groups. The top three of these 16 genes were RNPS1, AGTR1 and FNBP1L. A combined PPI network between the SS vs. HC and the NASH vs. HC groups was constructed and visualized (Fig. 4A). The dark green circles represent unique genes in the NASH vs. HC group. The yellow circles represent unique genes in the SS vs. $\mathrm{HC}$ group, and the red circles represent genes overlapping in the $\mathrm{SS}$ vs. HC and NASH vs. HC groups. 
A

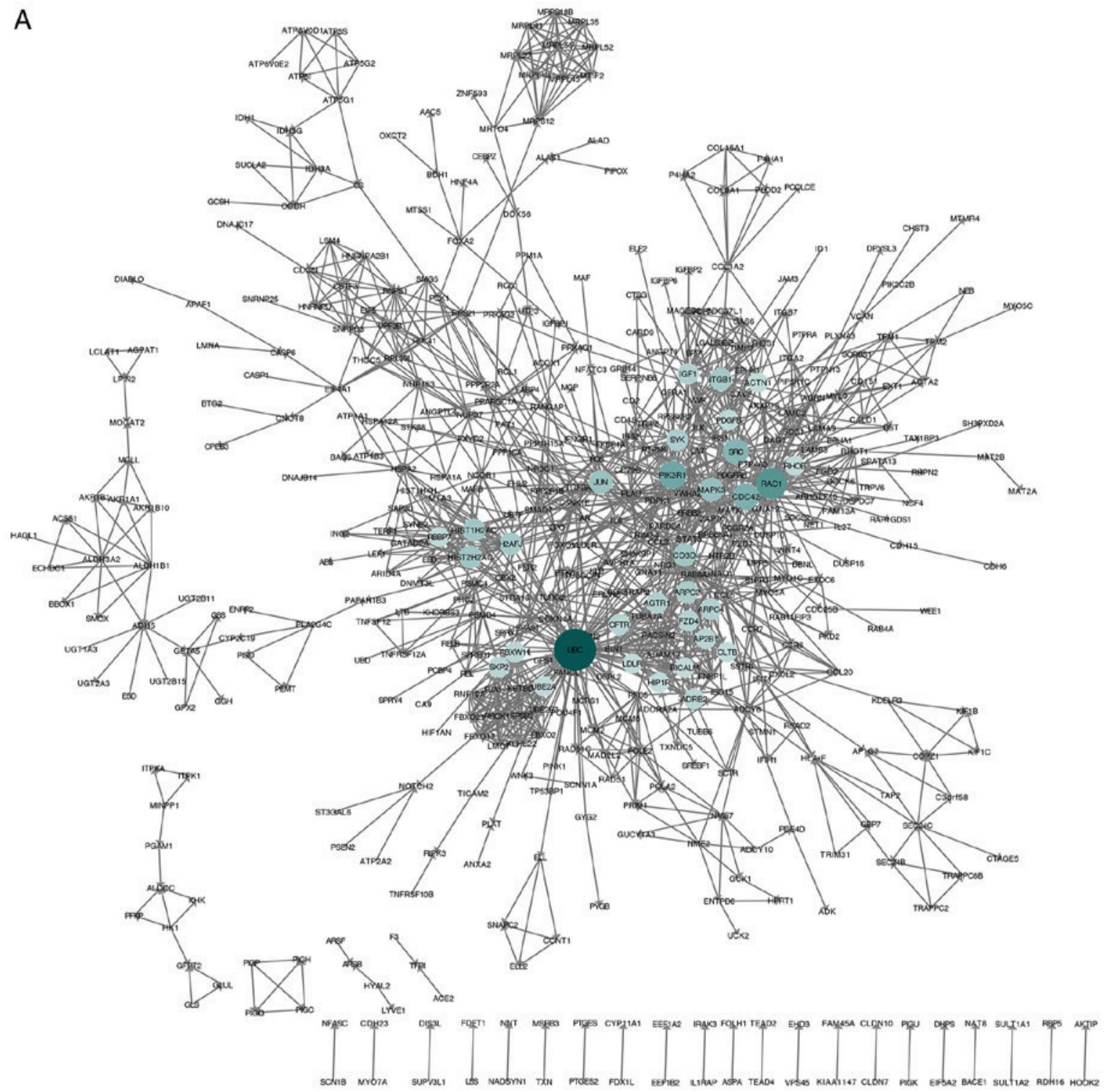

B
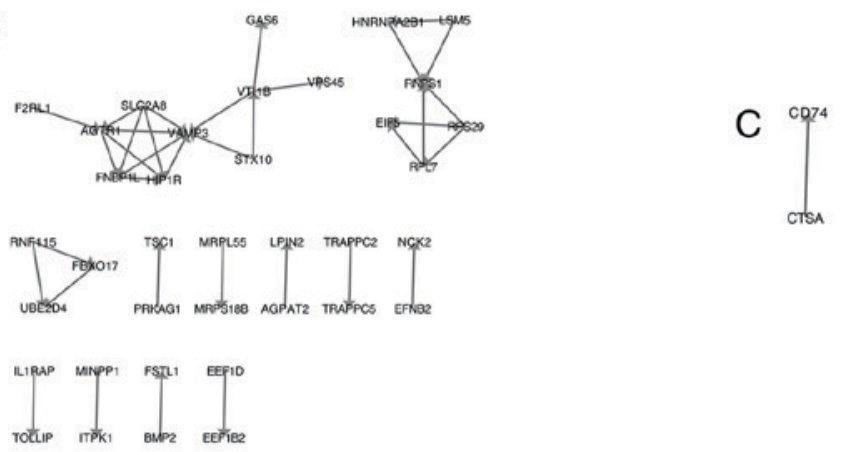

Figure 3. PPI network of identified DEGs constructed using Cytoscape software. (A) DEGs in the NASH vs. HC group. (B) DEGs in the SS vs. HC group. (C) DEGs in the NASH vs. SS group. The size and color depth of nodes represent the degree of one gene. The degree of one gene means the number of its interactions with other genes. The higher the degree of one gene, the more important it is in the PPI network. The edges represent the relationship between genes. DEG, differentially expressed gene; SS, steatosis; HC, healthy control; NASH, non-alcoholic steatohepatitis.

Furthermore, the top three modules of the combined PPI network were obtained using MCODE, a plug-in app for Cytoscape (Fig. 4B-D). KEGG pathway enrichment analysis was performed for the genes involved in the three modules using Webgestalt. The results revealed that 34 hub genes were involved in module one, and that they were significantly enriched in 'ubiquitin-mediated proteolysis' $\left(\mathrm{P}=1.86 \times 10^{-6}\right)$, 'endocytosis' $\left(\mathrm{P}=5.69 \times 10^{-6}\right)$ and 'Fc gamma receptor-mediated phagocytosis' $\left(\mathrm{P}=2.37 \times 10^{-3}\right)$. The gene with the highest degree in this module was UBC. A total of 19 hub genes were involved in module two, and they were significantly enriched in 'focal adhesion' $\left(\mathrm{P}=3.33 \times 10^{-16}\right)$, 'ECM-receptor interaction' 
A
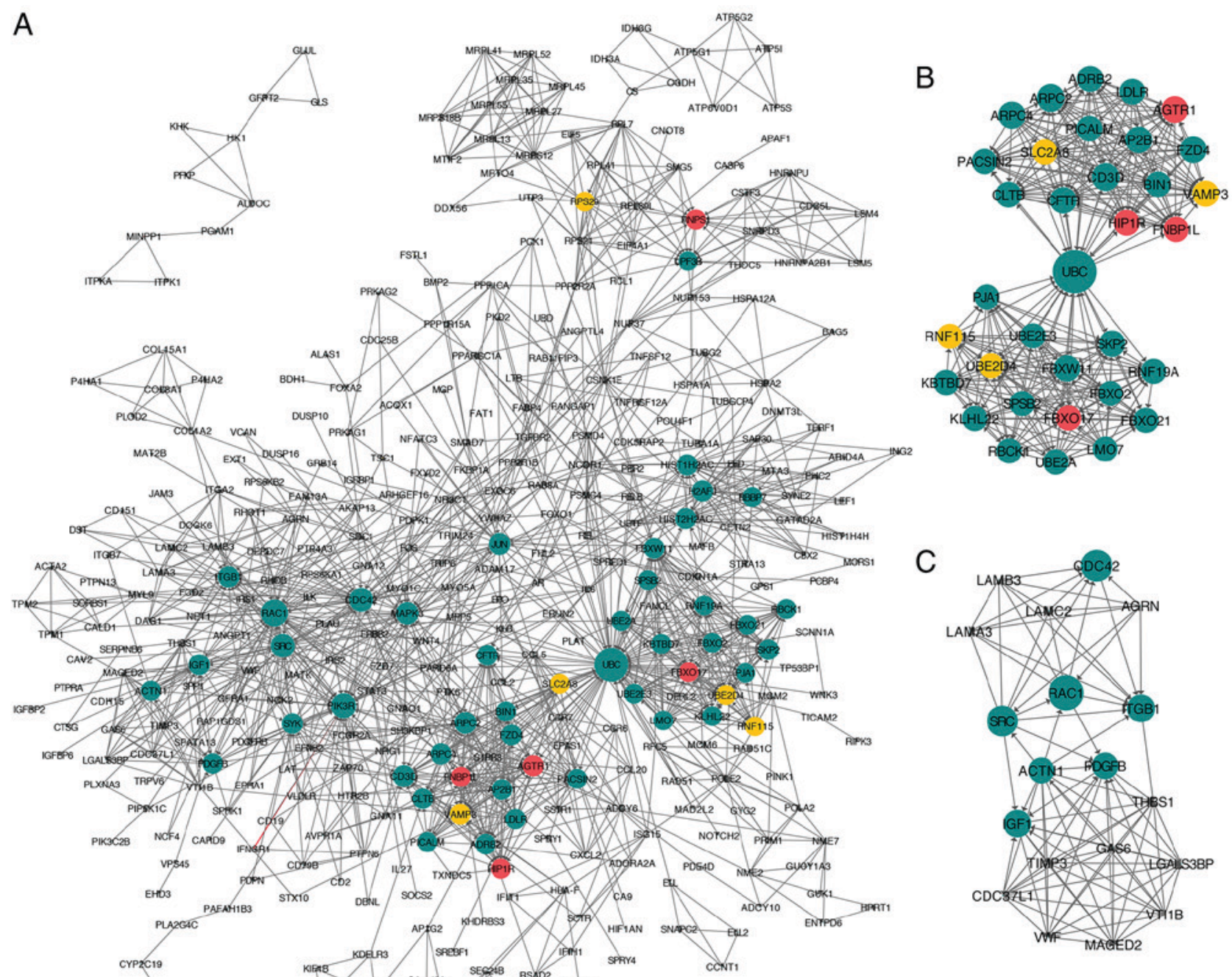

C
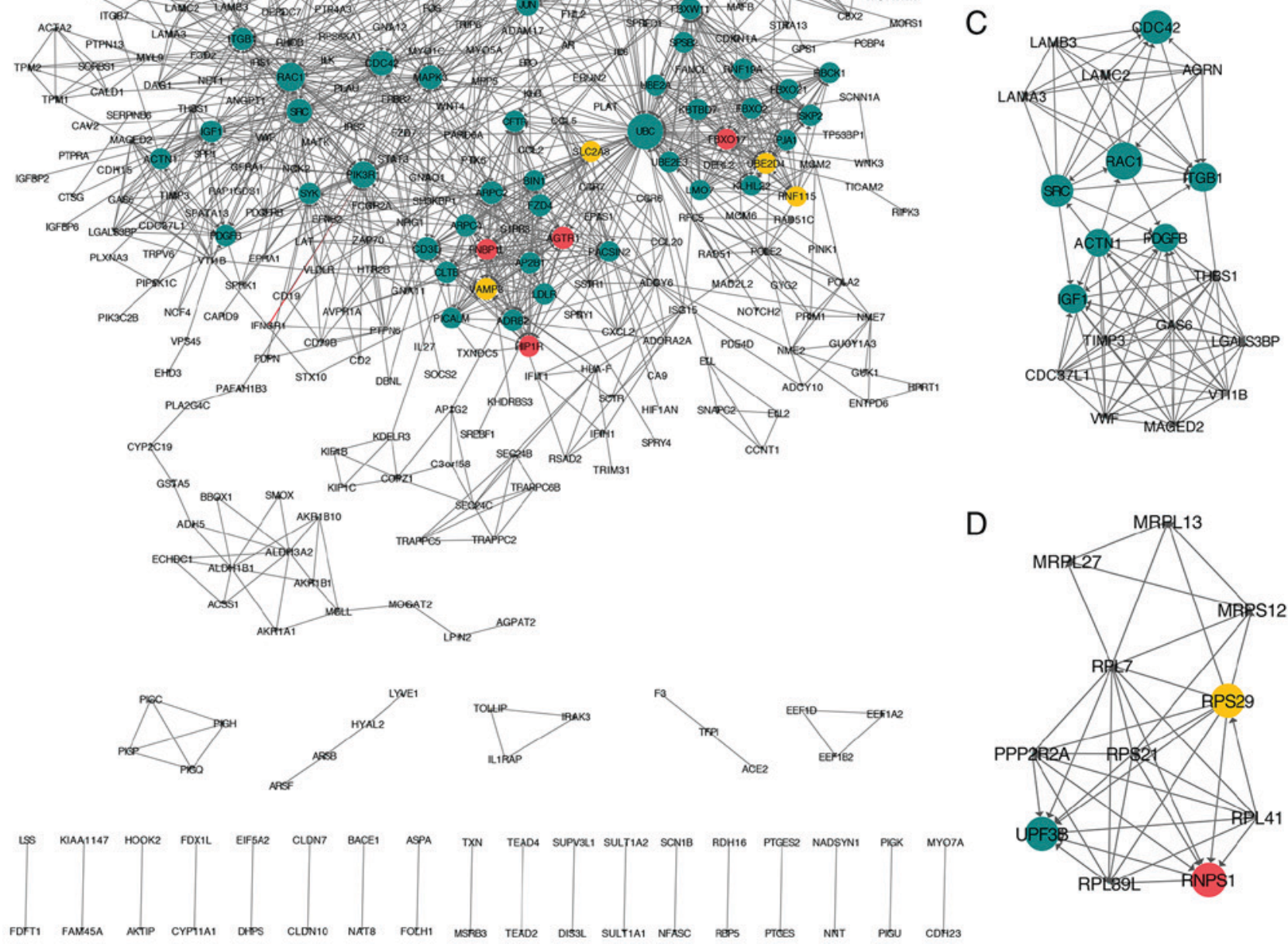

Figure 4. PPI network of identified DEGs constructed using Cytoscape software. The top three modules were obtained using MCODE. (A) PPI network. DEGs in the NASH vs. HC group are marked in dark green. DEGs in the SS vs. HC group are marked in yellow. DEGs that overlapped in the SS vs. HC and the NASH vs. HC groups are marked in red. The size of the node indicates the node degree of the genes. (B) Module (B) one, (C) two and (D) three of the PPI network. The top three modules were extracted from the network. PPI, protein-protein interaction; DEG, differentially expressed genes; HC, healthy control; SS, steatosis; NASH, non-alcoholic steatohepatitis; MCODE, multicontrast delayed enhancement.

$\left(\mathrm{P}=2.07 \times 10^{-10}\right)$ and 'phosphoinositide 3-kinase-protein kinase B signaling pathway' $\left(\mathrm{P}=9.49 \times 10^{-9}\right)$. The gene with the highest degree in module two was RACl. Furthermore, 11 hub genes were involved in module three, and they were significantly enriched in 'ribosome' $\left(\mathrm{P}=2.08 \times 10^{-10}\right)$, 'mRNA surveillance pathway' $\left(\mathrm{P}=2.22 \times 10^{-4}\right)$ and 'RNA transport'
$\left(\mathrm{P}=2.24 \times 10^{-2}\right)$. The gene with the highest degree in module three was RNPS1.

Furthermore, correlation analysis between the hub genes and diseases was performed with Webgestalt. The results revealed that the DEGs in module one were associated with hyperlipidemia $\left(\mathrm{P}=3.68 \times 10^{-4}\right)$ and bronchiectasis 
$\left(\mathrm{P}=8.53 \times 10^{-3}\right)$. The candidate drugs for the DEGs in module one were predominantly involved in the structural stabilization of the cytoskeleton. Additionally, the candidate miRNA targets for the DEGs were obtained using Webgestalt. The identified associated miRNAs were miR-101, miR-527 and miR-520F (Fig. 5A). In module two, the DEGs were associated with colitis $\left(\mathrm{P}=5.31 \times 10^{-6}\right)$, neoplasm invasiveness $\left(\mathrm{P}=3.4 \times 10^{-5}\right)$ and fibrosis $\left(\mathrm{P}=1.65 \times 10^{-3}\right)$. The drugs in module two were predominantly associated with oxygen binding and lipopeptide binding. The identified related miRNAs were miR-29A, miR-29B, miR-29C, miR-1 and miR-206 (Fig. 5B). In module three, the DEGs were associated with disease progression $\left(\mathrm{P}=1.78 \times 10^{-3}\right)$, anemia $\left(\mathrm{P}=8.53 \times 10^{-3}\right)$ and stomach neoplasms $\left(\mathrm{P}=8.65 \times 10^{-3}\right)$. The drugs in module three were predominantly associated with protein serine/threonine phosphatase activity. The identified related miRNAs were miR-218, miR-422B and miR-422A (Fig. 5C).

Validation of hub genes by $R T-q P C R$. To confirm the reliability of the identified hub genes, $\mathrm{LO} 2$ cells were treated with OA to induce lipid accumulation. RT-qPCR analysis was conducted to verify the expression levels of the identified genes. The results revealed that the majority of hub genes were differentially expressed in OA-treated cells (Fig. 6). RAC1, RNPS1 and UBC were significantly upregulated in OA-induced steatotic cells, compared with the control cells $(\mathrm{P}<0.05)$. AGTR1 was significantly downregulated in OA-induced steatotic cells, compared with the control cells $(\mathrm{P}<0.05)$. FNBP1L appeared to be upregulated in OA-induced steatotic cells, although the statistical difference was not significant $(\mathrm{P}=0.0807)$. Overall, the expression profiles of the hub genes were reasonably consistent between the bioinformatics analysis and RT-qPCR experiment.

\section{Discussion}

To reduce error and bias, two mRNA microarray datasets were obtained from the GEO database. A total of 190 DEGs were identified in the SS vs. HC group, 1,106 DEGs in the NASH vs. HC group and 34 DEGs in the NASH vs. SS group. Subsequently, a Venn diagram was utilized to identify the DEGs that may have a role specifically in NAFLD. FCGR2A and LARP1B were identified in all three groups (SS vs. HC, NASH vs. HC and NASH vs. SS). Thus, these genes may be considered to be specific diagnostic markers for NAFLD. It has been reported that certain genotypes of FCGR2A are associated with a more frequent occurrence of acute coronary syndromes, due to alterations in its interaction with C-reactive protein (21). Additionally, studies have demonstrated that FCGR2A and tyrosine-protein kinase Jak2 are associated with ulcerative colitis in Japanese and Korean populations $(22,23)$. Furthermore, emerging data has indicated the co-existence of NAFLD and inflammatory bowel disease (24). Thus, the aforementioned studies indicated that FCGR2A may be involved in organ damage in NAFLD. LARP1B is a paralogue of LARP1, which has not been widely studied in humans to the best of our knowledge.

GO and KEGG analyses were performed to examine the functional and biological interactions among the DEGs. GO and KEGG analysis revealed that the DEGs in the
SS vs. HC group were significantly enriched in 'cellular macromolecule biosynthetic process', 'regulation of RNA metabolic process', 'metabolic pathways' and 'cGMP-PKG signaling pathway'. It has been demonstrated that in the early stages of NAFLD, the principal pathophysiological alterations in the liver include insulin resistance and simple steatosis (25). Insulin resistance is closely associated with mitochondrial dysfunction, lipid peroxidation and energy imbalance, which eventually results in dysfunction and structural damage to cells and organs. Hepatic lipid metabolism disequilibrium initiated by insulin resistance, particularly in triglycerides, may result in lipid accumulation and steatosis, which triggers a series of cytotoxic events in the course of NAFLD (25).

The results of the GO and KEGG analyses for the SS vs. HC group revealed that alterations predominantly occurred in 'biosynthetic process', 'insulin signaling pathway' and 'metabolism', which may contribute to hepatocyte steatosis. In the NASH vs. HC group, the DEGs were significantly enriched in 'cellular response to chemical stimulus', 'response to organic substance', 'AMPK signaling pathway', 'insulin resistance' and 'FoxO signaling pathway'. In the NASH vs. SS group, the DEGs were significantly enriched in 'ECM organization', 'regulation of phosphate metabolic process' and 'ECM-receptor interaction'. Experimental observations have demonstrated that in intermediate and advanced stages of NAFLD, the principal pathophysiological alterations in the liver include oxidative stress and cytokine release (26). The anti-oxidative and anti-lipid peroxidation capacities of hepatocytes with steatosis are eradicated, which may result in inflammatory cell infiltration, inflammatory necrosis, hepatic fibrosis and even hepatocellular carcinoma (HCC) (26).

The AMPK signaling pathway participates in metabolic processes associated with glycolipids and has recently been regarded as a key research target for metabolic diseases. In oxidative stress, the AMPK signaling pathway may regulate autophagy by inducing protein phosphorylation, thus affecting energy and nutrient metabolism (27). One of the key transcription factors required for AMPK to be able to affect metabolism is FoxO1. A previous study reported that FoxO1 may regulate oxidative stress and insulin resistance by inducing the expression of gluconeogenic genes (28). The GO and KEGG terms identified in the present study may aid in the understanding of the underlying mechanisms of NAFLD.

Following the use of multiple screening measures, the top three genes, AGTR1, RNPS1 and FNBP1L, were obtained from the combined PPI network. AGTR1 has been hypothesized to mediate the major cardiovascular effects of angiotensin II (29). In diabetic nephropathy, angiotensin II may increase expression of AGTR1, leading to pronephrotic alterations (30). Activated AGTR1 may subsequently reduce the level of plasma adiponectin, and increase the activity of caspase 3/7 and cannabinoid receptor type 1 signaling in the kidney, leading to podocyte injury and diabetic glomerulopathy $(30,31)$. Furthermore, polymorphisms in AGTR1 may influence the risk of liver fibrosis in NAFLD (32). Taken together, these data indicate that AGTR1 may have an important role in the progression of NAFLD. 


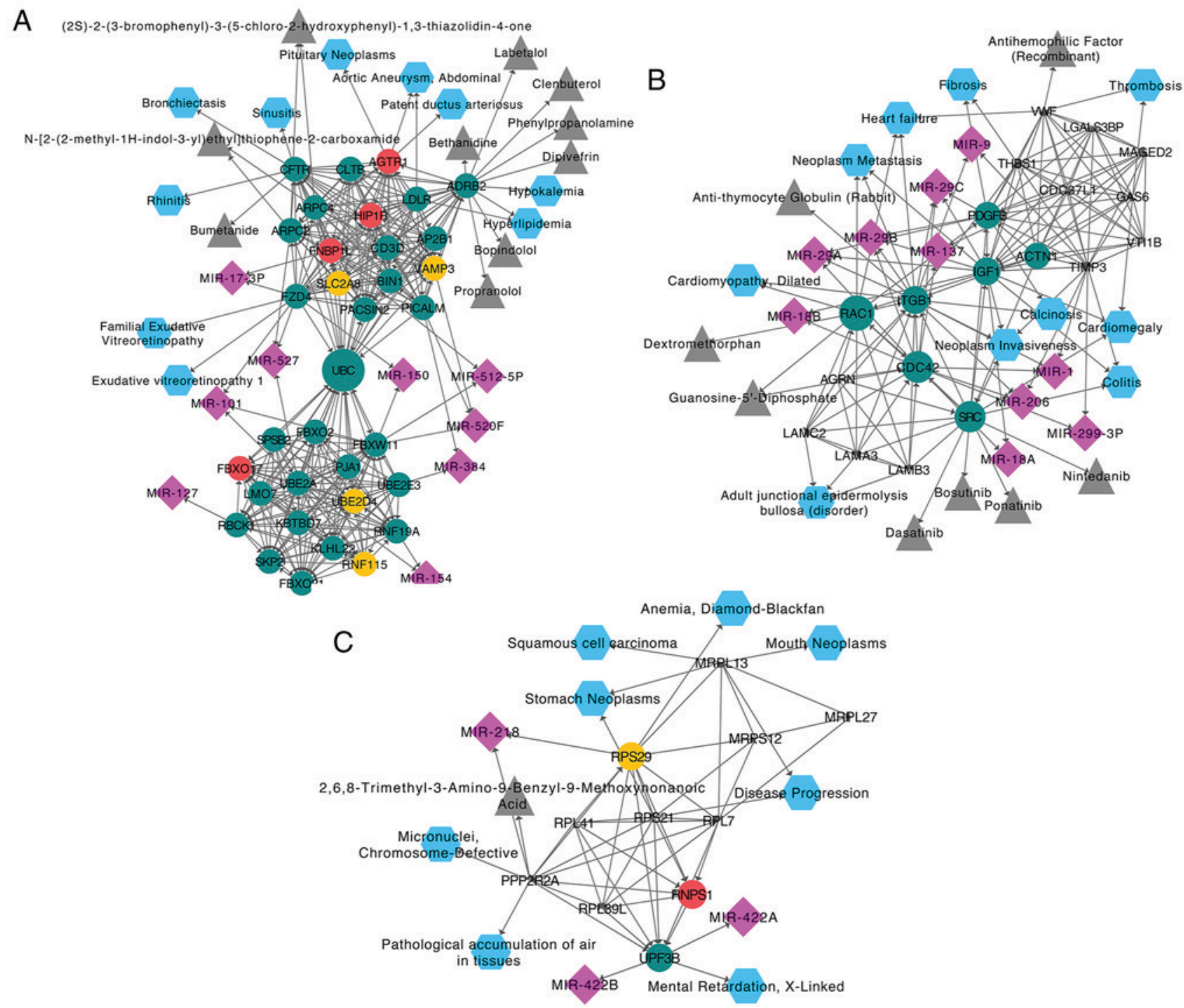

Figure 5. Hub genes in the top three modules. The associated miRNAs, drugs and diseases were identified by correlation analysis with Webgestalt. (A) Module one, (B) module two and (C) module three. miRNAs are marked with a purple diamond. Drugs are marked with a gray triangle. Diseases are marked with a blue hexagon. miRNA, microRNA.

RNPS1, a general activator of pre-mRNA splicing, is involved in mRNA nuclear export and mRNA surveillance (33). A previous study suggested that RNPS1 is associated with RNA processing and apoptosis, and is involved in programmed cell death (34). FNBP1L is able to bind to cell division control protein 42 (CDC42) and is thought to be essential for the autophagy of intracellular pathogens (35). The increased expression of FNBP1 L in breast cancer cells results in enhanced tumor metastasis and may be upregulated due to a loss of tumor suppressor p53 expression (36). However, no correlations have been reported among FNBP1L, RNPS1 and NAFLD.

Additionally, the top three modules were additionally extracted from the combined PPI network. Nodes with a higher degree were displayed in bigger circles. It was revealed that in module one, UBC was the core factor, with a degree of 81 . UBC is predominantly involved in protein degradation, the cell cycle and cell signaling pathway regulation $(37,38)$. A previous study reported that UBC exerts pathogenic effects in cardiac disease through regulation of the ubiquitin-proteasome system (39). However, no definite association has been identified between UBC and NAFLD. In module two, the identified core factor was RAC1, with a degree of 48. RAC1, a member of the Ras superfamily of small guanosine triphosphate-binding proteins, appears to regulate a number of cellular events, including cell growth, cytoskeletal reorganization and protein kinase activation $(40,41)$. It has been reported that RAC1 and CDC42 are principal activators in the saturated fatty acid-stimulated mitogen-activated protein kinase 8 pathway and cell death-associated pathogenesis of NAFLD (42). Thus, RAC1 may be the pathogenic contributor and progressive factor of NAFLD. In module three, the identified core factor was RNPS1 with a degree of 18 , which has been discussed above.

The mRNA expression levels of hub genes in OA-treated LO2 cells were additionally determined. The results revealed that the expression of AGTR1, RAC1, RNPS1 and UBC was significantly altered in OA-treated LO2 cells $(\mathrm{P}<0.05)$, which was consistent with the results of the bioinformatics analysis. The statistical difference in FNBP1L expression was not significant $(\mathrm{P}=0.0807)$, although its expression appeared to be 

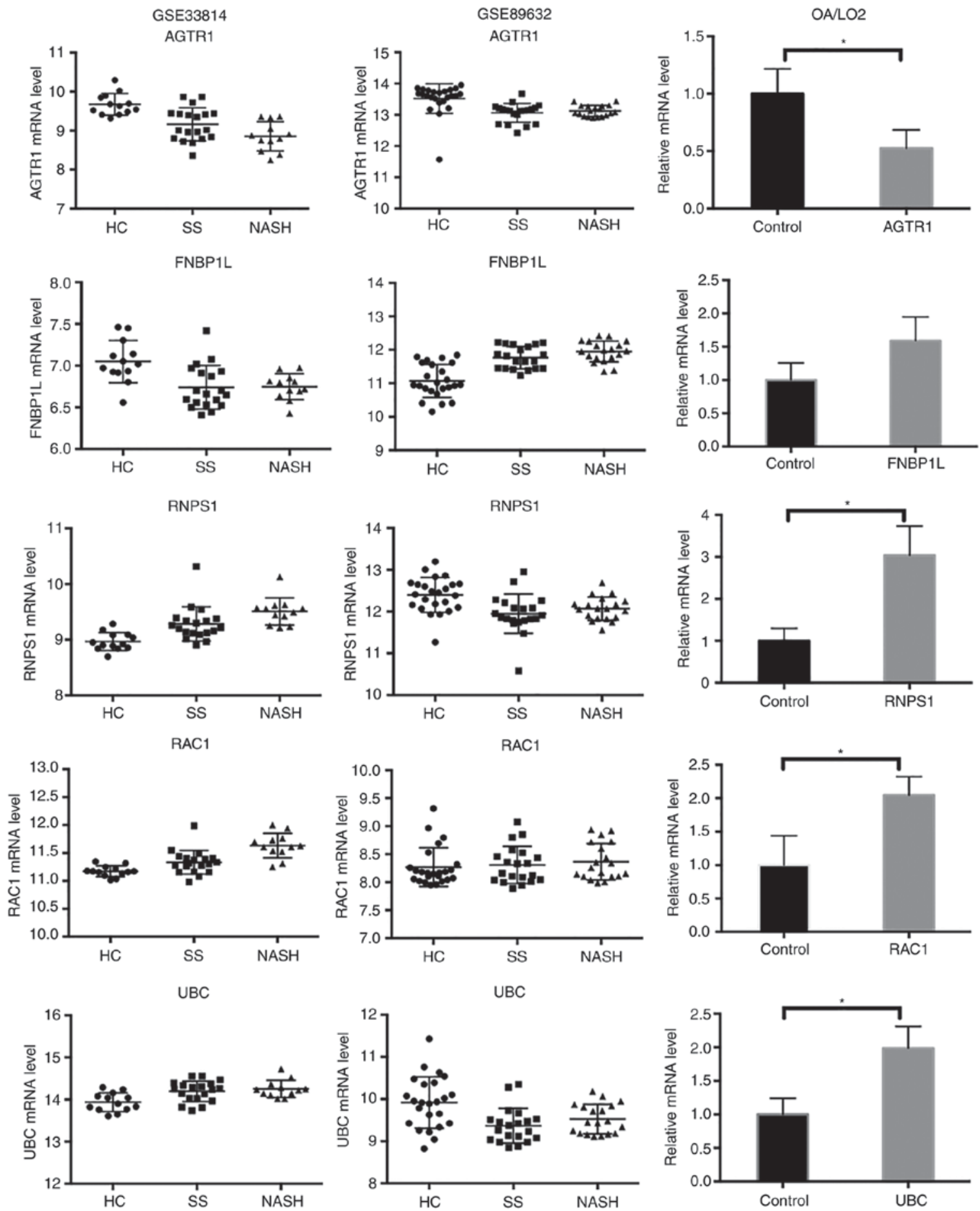

Figure 6. Analysis of hub genes with reverse transcription-quantitative polymerase chain reaction. The mRNA expression of AGTR1, FNBP1 L, RNPS1, RAC1 and UBC was analyzed in OA-treated cells and the control group. "P<0.05. AGTR1, type-1 angiotensin II receptor; FNBP1L, formin-binding protein 1-like; RNPS1, RNA-binding protein with serine-rich domain 1; RAC1, Ras-related C3 botulinum toxin substrate 1; UBC, polyubiquitin-C; OA, oleic acid.

upregulated in OA-treated cells. This may be due to several reasons. Firstly, gene chip screening for gene expression profiles is characterized by high throughput and a large scale, whereas PCR is suitable for studying alterations in the expression of a single gene. Furthermore, there may be differences between the preservation and detection of the samples in different research groups. Additionally, tissue samples are composed of numerous cell types and are therefore much more complex, whereas a cell line has a better homogeneity compared with a tissue sample. Finally, for a cell model, the different methods used to establish the cell model may affect the gene expression spectrum. Therefore, it is necessary to conduct in vivo experiments in the future to further verify the expression of the identified hub genes.

Due to a lack of NAFLD miRNA expression profile microarray datasets, the present study predicted the candidate miRNAs, drugs and diseases of NAFLD using Webgestalt. The key candidate disease in module one was hyperlipidemia, which 
was predominantly associated with ADRB2. The predicted candidate drugs were classified as antihypertensive drugs, which were additionally associated with ADRB2. ADRB2 has been reported to be involved in asthma and obesity $(43,44)$. ADRB2-knockout mice have attenuated phosphorylation of extracellular regulated protein kinase, which prevents diabetes mellitus-associated contractile dysfunction (45), indicating that ADRB2 may be a potential therapeutic target for diabetes mellitus-associated diseases of the cardiovascular system. The identified candidate miRNA was miR-101, which is linked to frizzled-4, F-box/WD repeat containing protein 11 and ubiquitin-conjugating enzyme E2 A. Previous studies have reported that miR-101 is associated with various tumor types and may regulate chemotherapeutic sensitivity in acute lymphoblastic leukemia $(46,47)$. However, there have been no studies focusing on the association between miR-101 and NAFLD, to the best of our knowledge.

The identified candidate diseases in module two were cardiac dysfunction and neoplasm progression, which were predominantly associated with RACl. The principal candidate drugs were anti-cancer drugs associated with proto-oncogene tyrosine-protein kinase Src (SRC). Studies have suggested that SRC is involved in the development and progression of certain cancer types $(48,49)$. The identified candidate miRNA was miR-29B, which is able to suppress the progression of tumors and prevent liver fibrosis, indicating that it may be an effective therapeutic target for NAFLD-associated fibrosis and HCC (50-52). Additionally, miR-29B was linked with CDC42 in module two, which in turn was associated with the aforementioned hub genes RAC1 and FNBP1L. The identified candidate diseases in module three were certain tumor types, which were predominantly associated with 39S ribosomal protein L13, mitochondrial, which functions in mitochondrial translation. The candidate drug was 2,6,8-trimethyl-3-amino -9-benzyl-9-methoxynonanoic acid. The identified candidate miRNA was miR-218, which is reported to inhibit tumor invasion (53). The candidate drug and miRNA in module three are associated with protein phosphatase 2 regulatory subunit $\mathrm{B} \alpha$, a subunit of protein phosphatase 2 that regulates protein kinase B signaling activity and is thought to regulate liver fibrosis in biliary atresia $(54,55)$. Taken together, the above results suggest that the identified drugs and miRNAs are candidate therapeutic or adjuvant targets in NAFLD.

In the present study, five hub genes and the associated functions and pathways of these genes were identified in NAFLD through integrated bioinformatics-based analysis and in vitro confirmation. However, there were certain limitations to the present analysis. There were certain discrepancies between the bioinformatics analysis results and the in vivo experimental model. Therefore, further verification of the expression of the identified hub genes, particularly FNBP1L, in vivo is required. Additionally, screening of the expression profile of miRNAs in NAFLD samples is necessary to obtain the mRNA-miRNA interaction networks. A higher number of subjects should also be recruited in future experiments in order to decrease deviation.

\section{Acknowledgements}

The authors acknowledge the assistance from the staff at the department of infectious disease in The First Affiliated
Hospital of Chongqing medical university for providing experimental site and technical help.

\section{Funding}

No funding was received.

\section{Availability of data and materials}

All data generated or analyzed during this study are included in this published article.

\section{Authors' contributions}

LL, HL and $\mathrm{XH}$ conceived and designed the experiments. LL, HL, YW and YHu performed the experiments. LL, YHe and QL acquired, analyzed and interpreted the data and wrote the paper.

\section{Ethics approval and consent to participate}

Not applicable.

\section{Consent for publication}

Not applicable.

\section{Competing interests}

The authors declare that they have no competing interests.

\section{References}

1. Lewis JR and Mohanty SR: Nonalcoholic fatty liver disease: A review and update. Dig Dis Sci 55: 560-578, 2010.

2. Starmann J, Fälth M, Spindelböck W, Lanz KL, Lackner C, Zatloukal K, Trauner M and Sültmann H: Gene expression profiling unravels cancer-related hepatic molecular signatures in steatohepatitis but not in steatosis. PLoS One 7: e46584, 2012.

3. Lonardo A, Byrne CD, Caldwell SH, Cortez-Pinto H and Targher G: Global epidemiology of nonalcoholic fatty liver disease: Meta-analytic assessment of prevalence, incidence, and outcomes. Hepatology 64: 1388-1389, 2016.

4. Arendt BM, Comelli EM, Ma DW, Lou W, Teterina A, Kim T, Fung SK, Wong DK, McGilvray I, Fischer SE and Allard JP: Altered hepatic gene expression in nonalcoholic fatty liver disease is associated with lower hepatic n- 3 and n- 6 polyunsaturated fatty acids. Hepatology 61: 1565-1578, 2015.

5. Sookoian S, Castaño G, Gianotti TF, Gemma C, Rosselli MS and Pirola CJ: Genetic variants in STAT3 are associated with nonalcoholic fatty liver disease. Cytokine 44: 201-206, 2008.

6. Min HK, Mirshahi F, Verdianelli A, Pacana T, Patel V, Park CG, Choi A, Lee JH, Park CB, Ren S and Sanyal AJ: Activation of the GP130-STAT3 axis and its potential implications in nonalcoholic fatty liver disease. Am J Physiol Gastrointest Liver Physiol 308: G794-G803, 2015.

7. Miele L, Beale G, Patman G, Nobili V, Leathart J, Grieco A, Abate M, Friedman SL, Narla G, Bugianesi E, Day CP and Reeves HL: The Kruppel-like factor 6 genotype is associated with fibrosis in nonalcoholic fatty liver disease. Gastroenterology 135 : 282-291.e1, 2008.

8. Seo YS, Kim JH, Jo NY, Choi KM, Baik SH, Park JJ, Kim JS, Byun KS, Bak YT, Lee CH, et al: PPAR agonists treatment is effective in a nonalcoholic fatty liver disease animal model by modulating fatty-acid metabolic enzymes. J Gastroenterol Hepatol 23: 102-109, 2008.

9. Sazci A, Ergul E, Aygun C, Akpinar G, Senturk O and Hulagu S: Methylenetetrahydrofolate reductase gene polymorphisms in patients with nonalcoholic steatohepatitis (NASH). Cell Biochem Funct 26: 291-296, 2008. 
10. Sun MY, Zhang L, Shi SL and Lin JN: Associations between Methylenetetrahydrofolate Reductase (MTHFR) Polymorphisms and Non-Alcoholic Fatty Liver Disease (NAFLD) risk: A meta-analysis. PLoS One 11: e0154337, 2016.

11. Edgar R, Domrachev M and Lash AE: Gene expression omnibus: NCBI gene expression and hybridization array data repository. Nucleic Acids Res 30: 207-210, 2002.

12. R Core Team: R: A Language and Environment for Statistical Computing. R Foundation for Statistical Computing, Vienna, 2011. http://www.R-project.org/.

13. Huang DW, Sherman BT, Tan Q, Collins JR, Alvord WG, Roayaei J, Stephens R, Baseler MW, Lane HC and Lempicki RA The DAVID gene functional classification tool: A novel biological module-centric algorithm to functionally analyze large gene lists. Genome Biol 8: R183, 2007.

14. Franceschini A, Szklarczyk D, Frankild S, Kuhn M, Simonovic M, Roth A, Lin J, Minguez P, Bork P, von Mering C and Jensen LJ: STRING v9.1: Protein-protein interaction networks, with increased coverage and integration. Nucleic Acids Res 41 (Database Issue): D808-D815, 2013.

15. Smoot ME, Ono K, Ruscheinski J, Wang PL and Ideker T: Cytoscape 2.8: New features for data integration and network visualization. Bioinformatics 27: 431-432, 2011.

16. Bandettini WP, Kellman P, Mancini C, Booker OJ, Vasu S, Leung SW, Wilson JR, Shanbhag SM, Chen MY and Arai AE: MultiContrast Delayed Enhancement (MCODE) improves detection of subendocardial myocardial infarction by late gadolinium enhancement cardiovascular magnetic resonance: A clinical validation study. J Cardiovasc Magn Reson 14: 83, 2012.

17. Zhang B, Kirov S and Snoddy J: WebGestalt: An integrated system for exploring gene sets in various biological contexts. Nucleic Acids Res 33 (Web Server Issue): W741-W748, 2005.

18. Wang B, Li L, Fu J, Yu P, Gong D, Zeng C and Zeng Z: Effects of long-chain and medium-chain fatty acids on apoptosis and oxidative stress in human liver cells with steatosis. J Food Sci 81: H794-H800, 2016.

19. An S, Zhao LP, Shen LJ, Wang S, Zhang K, Qi Y, Zheng J, Zhang XJ, Zhu XY, Bao R, et al: USP18 protects against hepatic steatosis and insulin resistance via its deubiquitinating activity. Hepatology 66: 1866-1884, 2017.

20. Livak KJ and Schmittgen TD: Analysis of relative gene expression data using real-time quantitative PCR and the 2(-Delta Delta C(T)) method. Methods 25: 402-408, 2001.

21. Raaz D, Herrmann M, Ekici AB, Klinghammer L, Lausen B, Voll RE, Leusen JH, van de Winkel JG, Daniel WG, Reis A and Garlichs CD: FcgammaRIIa genotype is associated with acute coronary syndromes as first manifestation of coronary artery disease. Atherosclerosis 205: 512-516, 2009.

22. Yang SK, Jung Y, Kim H, Hong M, Ye BD and Song K: Association of FCGR2A, JAK2 or HNF4A variants with ulcerative colitis in Koreans. Dig Liver Dis 43: 856-861, 2011.

23. Asano K, Matsushita T, Umeno J, Hosono N, Takahashi A, Kawaguchi T, Matsumoto T, Matsui T, Kakuta Y, Kinouchi Y, et al: A genome-wide association study identifies three new susceptibility loci for ulcerative colitis in the Japanese population. Nat Genet 41: 1325-1329, 2009.

24. Chandrashekaran V, Seth RK, Dattaroy D, Alhasson F, Ziolenka J, Carson J, Berger FG, Kalyanaraman B, Diehl AM and Chatterjee S: HMGB1-RAGE pathway drives peroxynitrite signaling-induced IBD-like inflammation in murine nonalcoholic fatty liver disease. Redox Biol 13: 8-19, 2017.

25. Shulman GI: Cellular mechanisms of insulin resistance. J Clin Invest 106: 171-176, 2000.

26. Kumar R, Prakash S, Chhabra S, Singla V, Madan K, Gupta SD, Panda SK, Khanal S and Acharya SK: Association of pro-inflammatory cytokines, adipokines \& oxidative stress with insulin resistance \& non-alcoholic fatty liver disease. Indian J Med Res 136: 229-236, 2012

27. Kim J, Kundu M, Viollet B and Guan KL: AMPK and mTOR regulate autophagy through direct phosphorylation of Ulk1. Nat Cell Biol 13: 132-141, 2011.

28. Valenti L, Rametta R, Dongiovanni P, Maggioni M, Fracanzani AL, Zappa M, Lattuada E, Roviaro G and Fargion S: Increased expression and activity of the transcription factor FOXO1 in nonalcoholic steatohepatitis. Diabetes 57: 1355-1362, 2008.

29. Plat AW, Stoffers HE, de Leeuw PW, van Schayck CP, Soomers FL, Kester AD, Aretz K and Kroon AA: The association between arterial stiffness and the angiotensin II type 1 receptor (A1166C) polymorphism is influenced by the use of cardiovascular medication. J Hypertens 27: 69-75, 2009.
30. Jourdan T, Szanda G, Rosenberg AZ, Tam J, Earley BJ, Godlewski G, Cinar R, Liu Z, Liu J, Ju C, et al: Overactive cannabinoid 1 receptor in podocytes drives type 2 diabetic nephropathy. Proc Natl Acad Sci USA 111: E5420-E5428, 2014.

31. Kagawa T, Takao T, Horino T, Matsumoto R, Inoue K, Morita T and Hashimoto K: Angiotensin II receptor blocker inhibits tumour necrosis factor-alpha-induced cell damage in human renal proximal tubular epithelial cells. Nephrology (Carlton) 13: 309-315, 2008.

32. Yoneda M, Hotta K, Nozaki Y, Endo H, Uchiyama T, Mawatari H, Iida H, Kato S, Fujita K, Takahashi H, et al: Association between angiotensin II type 1 receptor polymorphisms and the occurrence of nonalcoholic fatty liver disease. Liver Int 29: 1078-1085, 2009.

33. Mayeda A, Badolato J, Kobayashi R, Zhang MQ, Gardiner EM and Krainer AR: Purification and characterization of human RNPS1: A general activator of pre-mRNA splicing. EMBO J 18: 4560-4570, 1999.

34. Schwerk C, Prasad J, Degenhardt K, Erdjument-Bromage H, White E, Tempst P, Kidd VJ, Manley JL, Lahti JM and Reinberg D: ASAP, a novel protein complex involved in RNA processing and apoptosis. Mol Cell Biol 23: 2981-2990, 2003.

35. Huett A, Ng A, Cao Z, Kuballa P, Komatsu M, Daly MJ, Podolsky DK and Xavier RJ: A novel hybrid yeast-human network analysis reveals an essential role for FNBP1L in antibacterial autophagy. J Immunol 182: 4917-4930, 2009.

36. Chander H, Brien CD, Truesdell P, Watt K, Meens J, Schick C, Germain D and Craig AW: Toca-1 is suppressed by p53 to limit breast cancer cell invasion and tumor metastasis. Breast Cancer Res 16: 3413, 2014

37. Murray JI, Whitfield ML, Trinklein ND, Myers RM, Brown PO and Botstein D: Diverse and specific gene expression responses to stresses in cultured human cells. Mol Biol Cell 15: 2361-2374, 2004.

38. Ryu KT, Maehr R, Gilchrist CA, Long MA, Bouley DM, Mueller B, Ploegh HL and Kopito RR: The mouse polyubiquitin gene UbC is essential for fetal liver development, cell-cycle progression and stress tolerance. EMBO J 26: 2693-2706, 2007.

39. Xu J, Li Z, Ren X, Dong M, Li J, Shi X, Zhang Y, Xie W, Sun Z, Liu $X$ and Dai Q: Investigation of pathogenic genes in chinese sporadic hypertrophic cardiomyopathy patients by whole exome sequencing. Sci Rep 5: 16609, 2015.

40. Frasa MA, Maximiano FC, Smolarczyk K, Francis RE, Betson ME, Lozano E, Goldenring J, Seabra MC, Rak A, Ahmadian MR and Braga VM: Armus is a Racl effector that inactivates Rab7 and regulates E-cadherin degradation. Curr Biol 20: 198-208, 2010.

41. Oka T, Ihara S and Fukui Y: Cooperation of DEF6 with activated Rac in regulating cell morphology. J Biol Chem 282: 2011-2018, 2007.

42. Sharma M, Urano F and Jaeschke A: Cdc42 and Rac1 are major contributors to the saturated fatty acid-stimulated JNK pathway in hepatocytes. J Hepatol 56: 192-198, 2012.

43. Carroll CL, Stoltz P, Schramm CM and Zucker AR: Beta2-adrenergic receptor polymorphisms affect response to treatment in children with severe asthma exacerbations. Chest 135: 1186-1192, 2009.

44. Kao WT, Yen YC and Lung FW: The effects of beta2 adrenergic receptor gene polymorphism in lipid profiles. Lipids Health Dis 7: 20, 2008.

45. Wang Q, Liu Y,Fu Q, Xu B, Zhang Y, Kim S, Tan R, Barbagallo F, West T, Anderson E, et al: Inhibiting insulin-mediated $\beta 2$-adrenergic receptor activation prevents diabetes-associated cardiac dysfunction. Circulation 135: 73-88, 2017.

46. Huang S, Yang Z, Ma Y, Yang Y and Wang S: miR-101 enhances cisplatin-induced DNA damage through decreasing nicotinamide adenine dinucleotide phosphate levels by directly repressing Tp53-induced glycolysis and apoptosis regulator expression in prostate cancer cells. DNA Cell Biol 36: 303-310, 2017.

47. Qian L, Zhang W, Lei B, He A, Ye L, Li X and Dong X: MicroRNA-101 regulates T-cell acute lymphoblastic leukemia progression and chemotherapeutic sensitivity by targeting Notch1. Oncol Rep 36: 2511-2516, 2016.

48. Jin C, Zhang X, Sun M, Zhang Y, Zhang G and Wang B: Clinical implications of the coexpression of SRC1 and NANOG in HER-2-overexpressing breast cancers. Onco Targets Ther 9: 5483-5488, 2016.

49. Laschi M, Bernardini G, Geminiani M, Manetti F, Mori M, Spreafico A, Campanacci D, Capanna R, Schenone S, Botta M and Santucci A: Differentially activated Src kinase in chemo-naïve human primary osteosarcoma cells and effects of a Src kinase inhibitor. Biofactors 43: 801-811, 2017. 
50. Zhu K, Liu L, Zhang J, Wang Y, Liang H, Fan G, Jiang Z, Zhang CY, Chen X and Zhou G: MiR-29b suppresses the proliferation and migration of osteosarcoma cells by targeting CDK6. Protein Cell 7: 434-444, 2016.

51. Fang JH, Zhou HC, Zeng C, Yang J, Liu Y, Huang X, Zhang JP, Guan XY and Zhuang SM: MicroRNA-29b suppresses tumor angiogenesis, invasion, and metastasis by regulating matrix metalloproteinase 2 expression. Hepatology 54: 1729-1740, 2011.

52. Wang J, Chu E, Chen H, Man K, Go M, Huang X, Lan H, Sung J and Yu J: microRNA-29b prevents liver fibrosis by attenuating hepatic stellate cell activation and inducing apoptosis through targeting PI3K/AKT pathway. Oncotarget 6: 7325-7338, 2015.

53. Zhang X, Dong J, He Y, Zhao M, Liu Z, Wang N, Jiang M, Zhang Z, Liu G, Liu H, et al: miR-218 inhibited tumor angiogenesis by targeting ROBO1 in gastric cancer. Gene 615: 42-49, 2017.
54. Dong R, Zheng Y, Chen G, Zhao R, Zhou Z and Zheng S: miR-222 overexpression may contribute to liver fibrosis in biliary atresia by targeting PPP2R2A. J Pediatr Gastroenterol Nutr 60: 84-90, 2015.

55. Kuo YC, Huang KY, Yang CH, Yang YS, Lee WY and Chiang CW: Regulation of phosphorylation of Thr-308 of Akt, cell proliferation, and survival by the B55alpha regulatory subunit targeting of the protein phosphatase $2 \mathrm{~A}$ holoenzyme to Akt. J Biol Chem 283: 1882-1892, 2008.

This work is licensed under a Creative Commons Attribution-NonCommercial-NoDerivatives 4.0 International (CC BY-NC-ND 4.0) License. 